\title{
Aldolase A and carbonic anhydrase III, two new candidate biomarkers versus acknowledged parameters in the context of crushing syndrome
}

\author{
Iulian Constantin CREANGA ${ }^{1,2}$, Alexandra RIPSZKY TOTAN², Daniela MIRICESCU², \\ Iulia-Ioana STANESCU-SPINU², Maria GREABU² \\ 1"Prof. Dr. Dimitrie Gerota” Emergency Hospital, Bucharest, Romania \\ ${ }^{2}$ Department of Biochemistry, Faculty of Dental Medicine, \\ "Carol Davila" University of Medicine and Pharmacy, Bucharest, Romania
}

\begin{abstract}
Introduction. Crushing syndrome can affect all structures of a limb and can cause unique or complex injuries. The clinical picture of the injuries is nonspecific and extremely varied, and is not characteristic of the mechanism that generated it. The aim of our study was to correlate the changes of ALD A (aldolase A) and CAIII (carbonic anhydrase III) in the appearance and evolution of the crushing syndrome.

Material and methods. The present study included 22 patients with crushing syndrome. The control group consisted of 20 healthy subjects. IL-6 (interleukin-6), CK (creatine kinase), ALD A and CAIII blood levels were determined simultaneously from all the participants to the study. Statistical analysis was performed using IBM SPSS Statistics 25 and Microsoft Office Excel / Word 2013.

Results. Our results showed significantly increased levels of ALD A, CAIII, IL-6 and CK in patients with crushing syndrome. However, no statistically significant correlations could be found between the studied biomarkers.

Discussions. So far, in the medical literature, very few studies focused on the correlation between the occurrence of crushing syndrome and changes in ALD A and CA III levels.

Conclusions. Our study showed that ALD A and CAIII may become important biomarkers for the rapid diagnosis of crushing syndrome in the future.
\end{abstract}

Keywords: crushing syndrome, aldolase A, carbonic anhydrase III, trauma

\section{INTRODUCTION}

Crushing syndrome can affect all structures of a limb and can cause unique or complex injuries. The clinical picture of the injuries is nonspecific and extremely varied, and is not characteristic of the mecha- nism that generated it. The clinical picture is expressed according to the extent to which each of these structures has been affected. It has been shown that the link between the onset of crushing syndrome and the change in biochemical markers can be used for prognostic purposes of the evolution of crushing syndrome. 
Among others, the concentration of skeletal muscle enzymes and proteins in serum or plasma are used to evaluate the functional status of muscle tissue, which can vary widely in both pathological and physiological conditions [1].

Aldolase A (ALD A) is an enzyme found in a variety of tissues, and although different expressions of this parameter can be found in many disorders, such as skeletal and inflammatory muscle disease, polymyositis, dermatomyositis, and in muscular dystrophy, few publications have reported variations in the serum levels of this marker in the crush syndrome context.

Carbonic anhydrase III (CAIII) is a member of the carbonic anhydrase (CA) family, which is abundantly expressed in skeletal muscles, liver and adipocytes [2-4]. CAIII plays an essential role in regulating intracellular $\mathrm{pH}$ and maintaining the acid-base balance. It also has an antioxidant role in energy metabolism, involved in the appearance and development of fatigue [2-4].

When subjected to severe injuries, skeletal muscles can hardly benefit from full functional restoration, therefore, early diagnosis of muscle injuries is very important for the therapeutic decisions in order to insure the full recovery of injured muscles. Muscle injuries trigger changes in terms of physical, physiological and biochemical aspects. However, the metabolic disturbances in the injured muscle are illustrated by the release in the extracellular matrix of specific biomarkers, such as: muscle proteins -myoglobin, troponin and enzymes - creatine kinase, the $\mathrm{MM}$ and $\mathrm{MB}$ isoenzymes (CK-MM; CK-MB), lactate dehydrogenase (LDH), aspartate aminotransferase (AST), and, the very little studied, aldolase (ALD A) and carbonic anhydrase III (CAIII). Quantifying these parameters blood levels may contribute substantially to muscle status evaluation.

\section{AIM}

The aim of our study was to identify the possible significant correlations between the acknowledged, but not very specific, muscle parameters (CK, IL-6) and two new possible candidates as biomarkers, ALD A and CA III, in the crushing syndrome context, in order to substantially improve the existing diagnostic and therapeutic protocols.

\section{MATERIAL AND METHODS}

The present study included the research carried out during the doctoral internship, performed on a group of patients, in which we aimed to identify a possible link between biochemical markers dedicated to muscle damage and ALD A, CA III, both by bibliographic research and quantitative analysis and statistics of some parameters of interest. All the participants, patients and controls, provided the informed consent to participate in the study. This study was approved by the Ethics Committee of the Emergency Clinical Hospital (SCUB), Bucharest, Romania (6885/12.07.2021).

The study included patients who have suffered a trauma due to a high-energy traumatic agent and patients who developed crush syndrome as a result of interaction with a medium energy, but with a prolonged duration of action on tissues.

Exclusion criteria from the study were: the presence of acute infectious diseases; the existence of a chronic infectious disease; a heart attack in the last 4 weeks; muscle necrosis or muscle stress of non-traumatic etiology; active cancers with damage to organs or soft tissues; the presence of another autoimmune disease with damage to tissues and organs (myocyte destruction - eg. systemic lupus erythematosus, rheumatoid arthritis).

Blood samples collection and serum samples preparation for all analyzed parameters (IL-6, CK, ALD A and CA III) were performed simultaneously from a lot of 42 subjects: 22 patients who have suffered a trauma due to a high-energy traumatic agent (patients who developed crush syndrome as a result of interaction with a medium energy but with a prolonged duration of action on tissues), and 20 healthy subjects without any pathology of traumatic etiology that were included in the control group. The average age of the participants in the two groups was: $40+/-7$ years for the patients who suffered a high energy trauma, and $37+/-11$ years for the subjects included in the control group.

Measurement of serum biomarkers was performed using freshly frozen samples that were maintained at $-80^{\circ} \mathrm{C}$ until the time of assay. For determining the levels of ALD A and CA III we used Cusabio assay kit (Wuhan Hi-tech Medical Devices Park - China) that was performed on semiautomatic immunoassay system STAT FAX 303 PLUS (Awareness Technologies). Serum IL-6 levels have been measured on a chemiluminescence automatic analyzer, Maccura1200 Plus (China). For the serum CK levels we have used a biochemistry automatic analyzer, A25 - Biosystems, Spain.

Statistical analysis was performed using IBM SPSS Statistics 25 and Microsoft Office Excel / Word 2013. Quantitative variables were tested for distribution using the Shapiro-Wilk test and were expressed as means with standard deviations or medians with interpercentile intervals. The independent quantitative variables with non-parametric distribution were tested using the Mann-Whitney $U$ test and the independent quantitative variables with parametric distribution were tested using the Student's t-test (the existing correlations between them were tested using the Pearson correlation coefficient). 


\section{RESULTS}

Our results for the four studied parameters are summarized in Tables 1-6 and Figures 1-7.

TABLE 1. Mean value of the parameters evaluated in the study

\begin{tabular}{|c|c|c|c|}
\hline \multicolumn{2}{|c|}{ Parameter: } & \multirow{2}{*}{$\begin{array}{c}\text { Mean } \pm \text { SD } \\
\begin{array}{c}37.48 \pm 19.31 \mathrm{ng} / \\
\mathrm{ml}\end{array}\end{array}$} & $\begin{array}{c}p^{*} \\
(p<0.001 * * *)\end{array}$ \\
\hline CA III & $\begin{array}{c}\text { Control lot } \\
\left(p=0.001^{* *}\right)\end{array}$ & & \multirow{8}{*}{$<0.001$} \\
\hline & $\begin{array}{c}\text { Study lot } \\
\left(p=0.316^{* *}\right)\end{array}$ & $\begin{array}{c}154.06 \pm 18.97 \\
\mathrm{ng} / \mathrm{ml}\end{array}$ & \\
\hline \multirow[t]{2}{*}{ ALD A } & $\begin{array}{c}\text { Control lot } \\
\left(p=0.664^{* *}\right)\end{array}$ & $\begin{array}{c}961.82 \pm 165.7 \\
\mathrm{pg} / \mathrm{ml}\end{array}$ & \\
\hline & $\begin{array}{c}\text { Study lot } \\
\left(p=0.414^{* *}\right)\end{array}$ & $\begin{array}{c}3105 \pm 849.81 \mathrm{pg} / \\
\mathrm{ml}\end{array}$ & \\
\hline \multirow[t]{2}{*}{ IL-6 } & Control lot & $2.2 \pm 0.6 \mathrm{pg} / \mathrm{ml}$ & \\
\hline & Study lot & $8.52 \pm 1.2 \mathrm{pg} / \mathrm{ml}$ & \\
\hline \multirow[t]{2}{*}{ CK } & Control lot & $218.6 \pm 69 \mathrm{U} / \mathrm{l}$ & \\
\hline & Study lot & $1286.5 \pm 561.6 \mathrm{U} / \mathrm{I}$ & \\
\hline
\end{tabular}

Our results showed significantly increased levels of ALD A in patients with trauma $(3105 \pm 849.81 \mathrm{pg} / \mathrm{ml})$ compared to the control group $961.82 \pm 165.7 \mathrm{pg} / \mathrm{ml}$ ) $(p<0.001)$ (Table 1, Figure 1).

According to the Student's test, patients in the study group had significantly higher CA III levels (1540.6 $\pm 18.97 \mathrm{ng} / \mathrm{ml}$ ) compared to patients in the control group $(37.48 \pm 19.31 \mathrm{ng} / \mathrm{ml})(\mathrm{p}<0.001)$ (Table 1 , Figure 2).

TABLE 2. Correlation between carbonic anhydrase III and aldolase A levels in the study group

\begin{tabular}{|l|l|}
\hline Corelation & $\mathbf{p}^{*}$ \\
\hline CA III x ALD A & $0.198, R=0.285$ \\
\hline
\end{tabular}

*Pearson correlation coefficient

The data presented in Table 2 and Figure 3 shows that the is no significant correlation between CAIII and ALD A levels in the study group $(p=0.198, R=0.285)$.

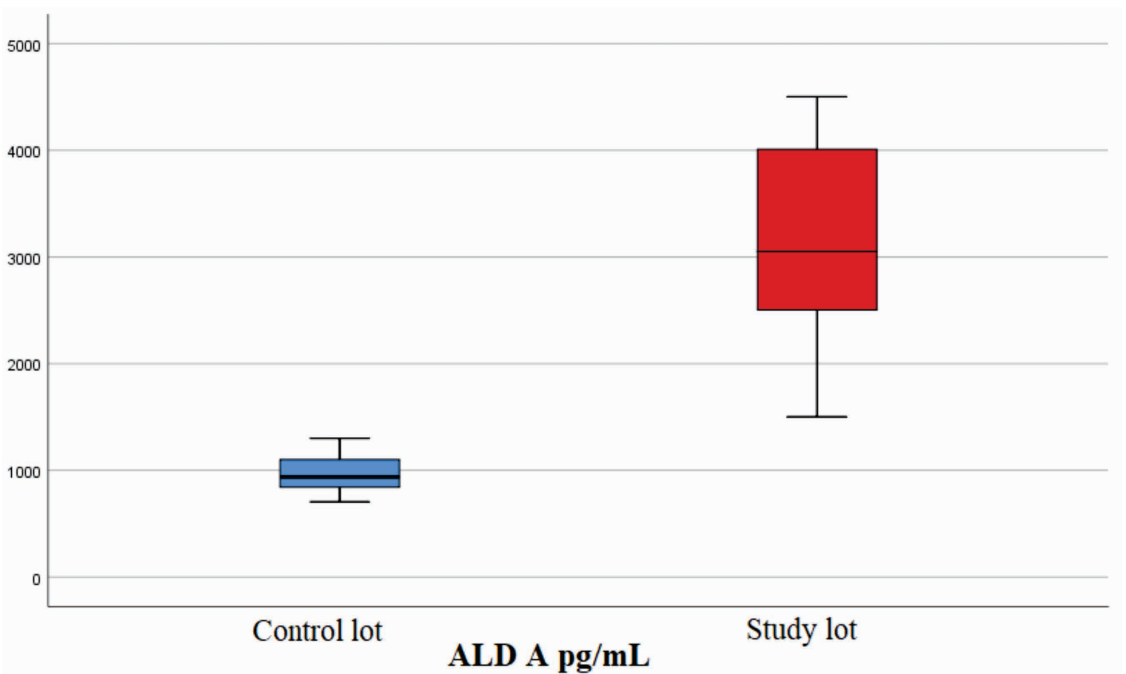

FIGURE 1. Comparison of ALD A levels between the 2 groups

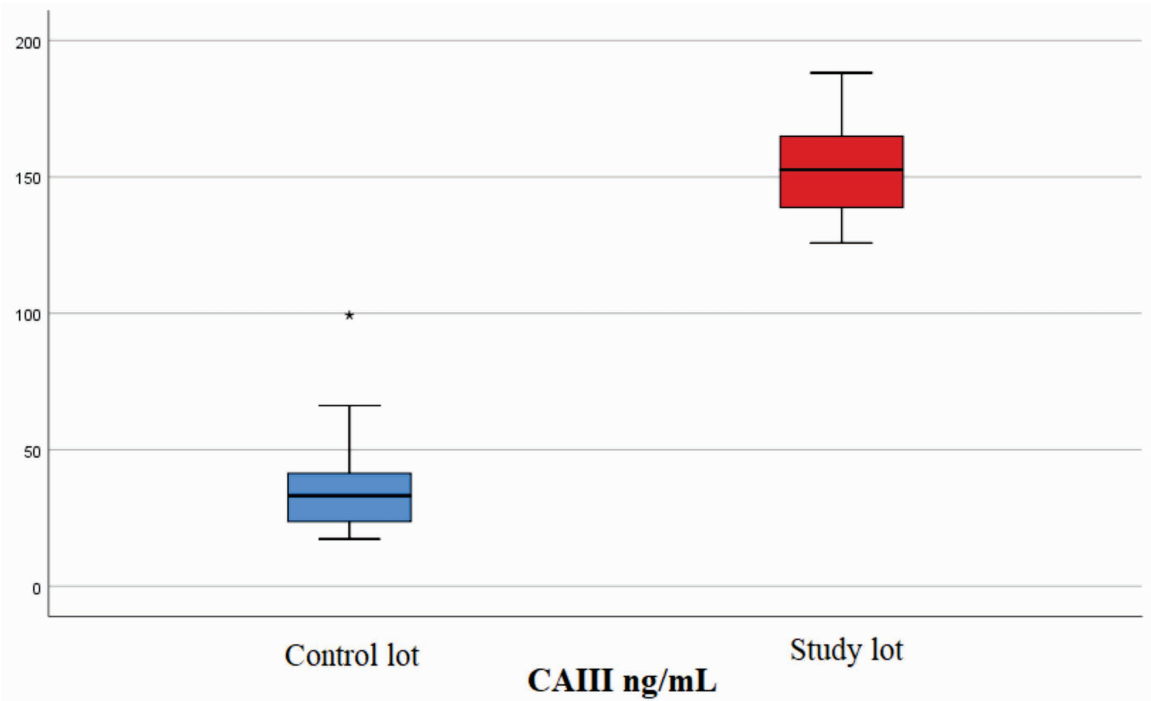

FIGURE 2. CAIIl levels in patients with trauma compared to the control group 


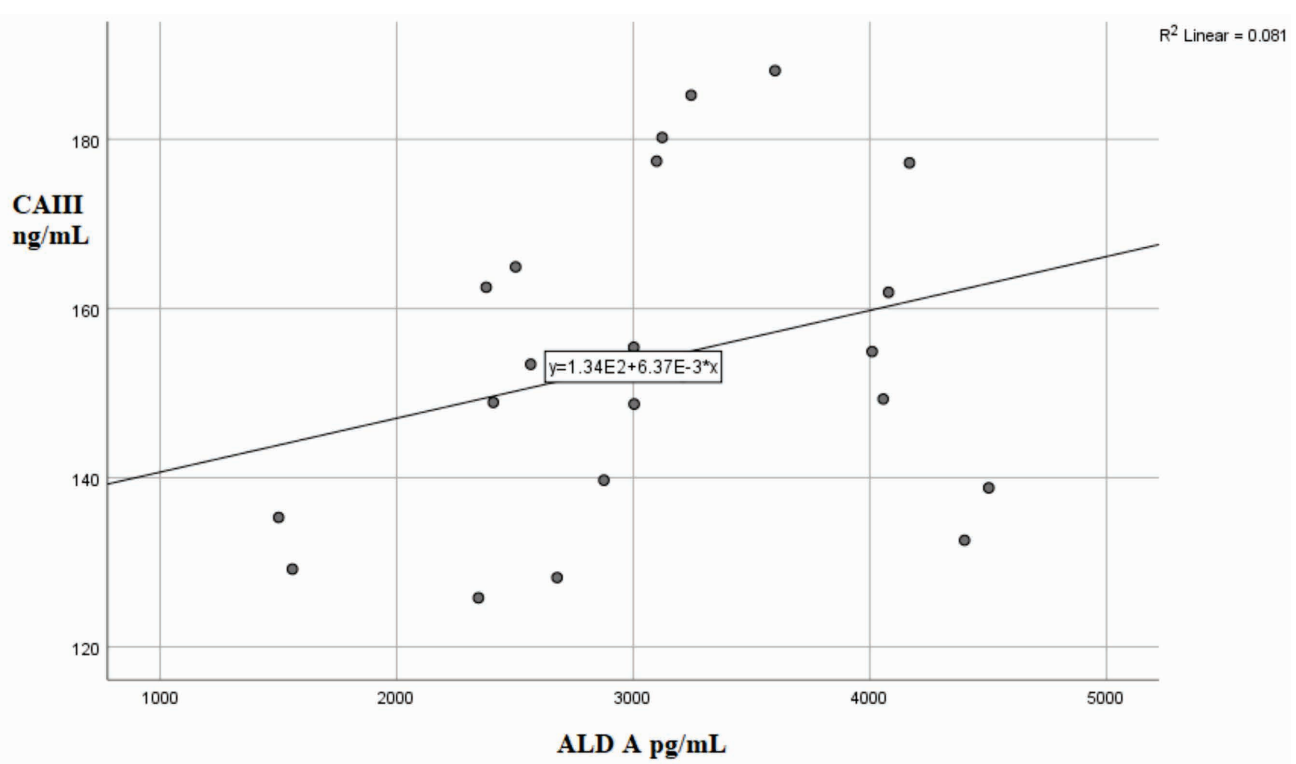

FIGURE 3. Correlation between carbonic anhydrase III and aldolase A levels in crushing syndrome patients

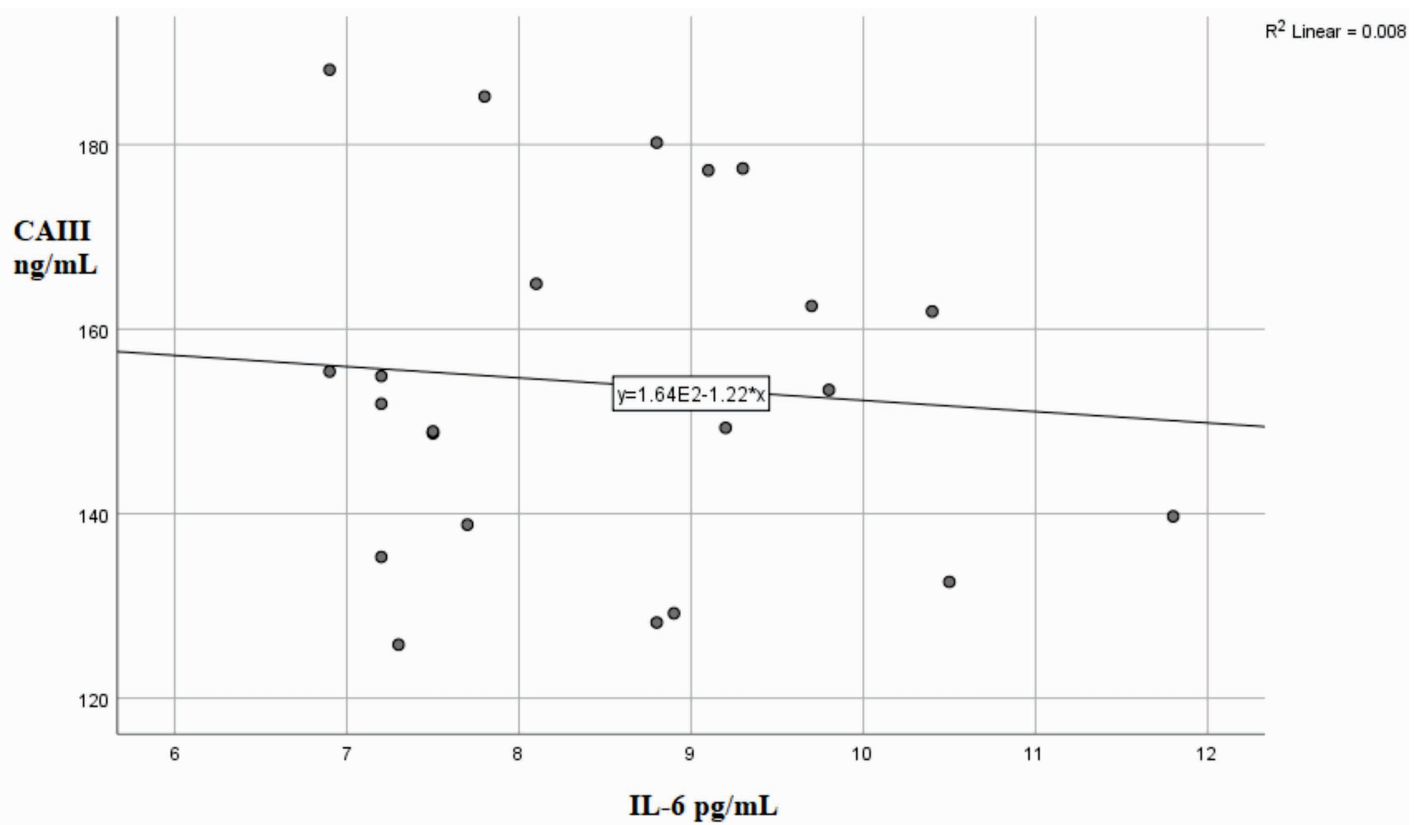

FIGURE 4. Correlation between carbonic anhydrase III values and IL-6 valuesin the study group

TABLE 3. Correlation between carbonic anhydrase III and IL-6 levels in the study group

\begin{tabular}{|l|l|}
\hline Corelation & $\mathbf{p}^{*}$ \\
\hline AC III x IL-6 & $0.701, R=-0.087$ \\
\hline
\end{tabular}

*Pearson correlation coefficient

Table 3 and Figure 4 present the correlation between carbonic anhydrase III values and IL-6 levels in the study group. The observed correlation between $\mathrm{AC}$ III and IL- 6 was observed to be insignificant ( $p=0.701$, $\mathrm{R}=-0.087$ ), so in patients with crushing syndrome, no significant association was found between these two variables.
TABLE 4. Correlation between carbonic anhydrase III and CK levels in trauma patients

\begin{tabular}{|l|l|}
\hline Corelation & $\mathbf{p}^{*}$ \\
\hline AC III x CK & $0.654, \mathrm{R}=0.189$ \\
\hline
\end{tabular}

*Pearson correlation coefficient

The data in Table 4 and Figure 5 represent the correlation between CAIII and CK values in the study group. The observed correlation between CAIII and CK was statistically insignificant $(p=0.654, R=0.189)$. 


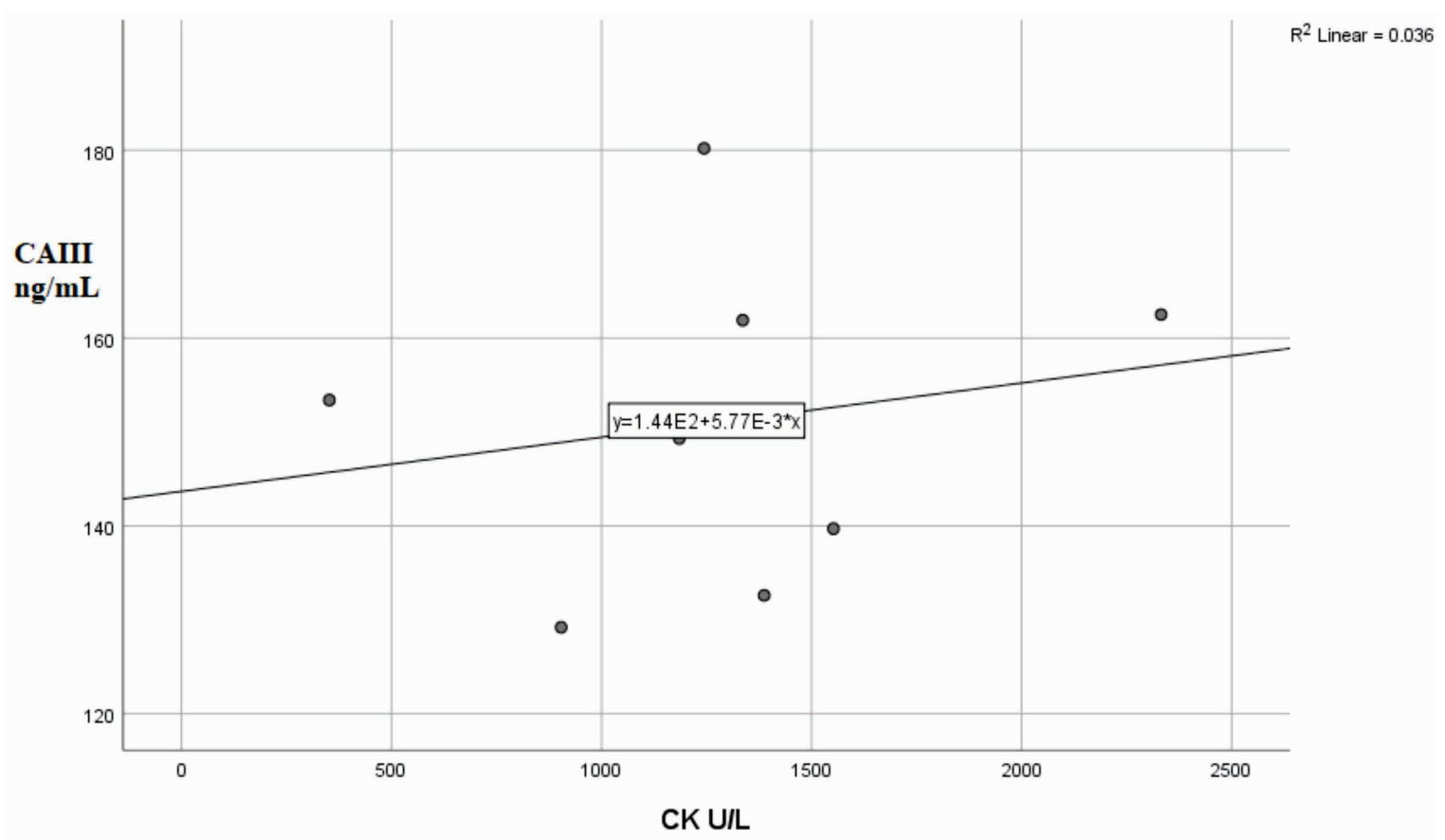

FIGURE 5. Correlation between CAIIl and CK levels in the study group

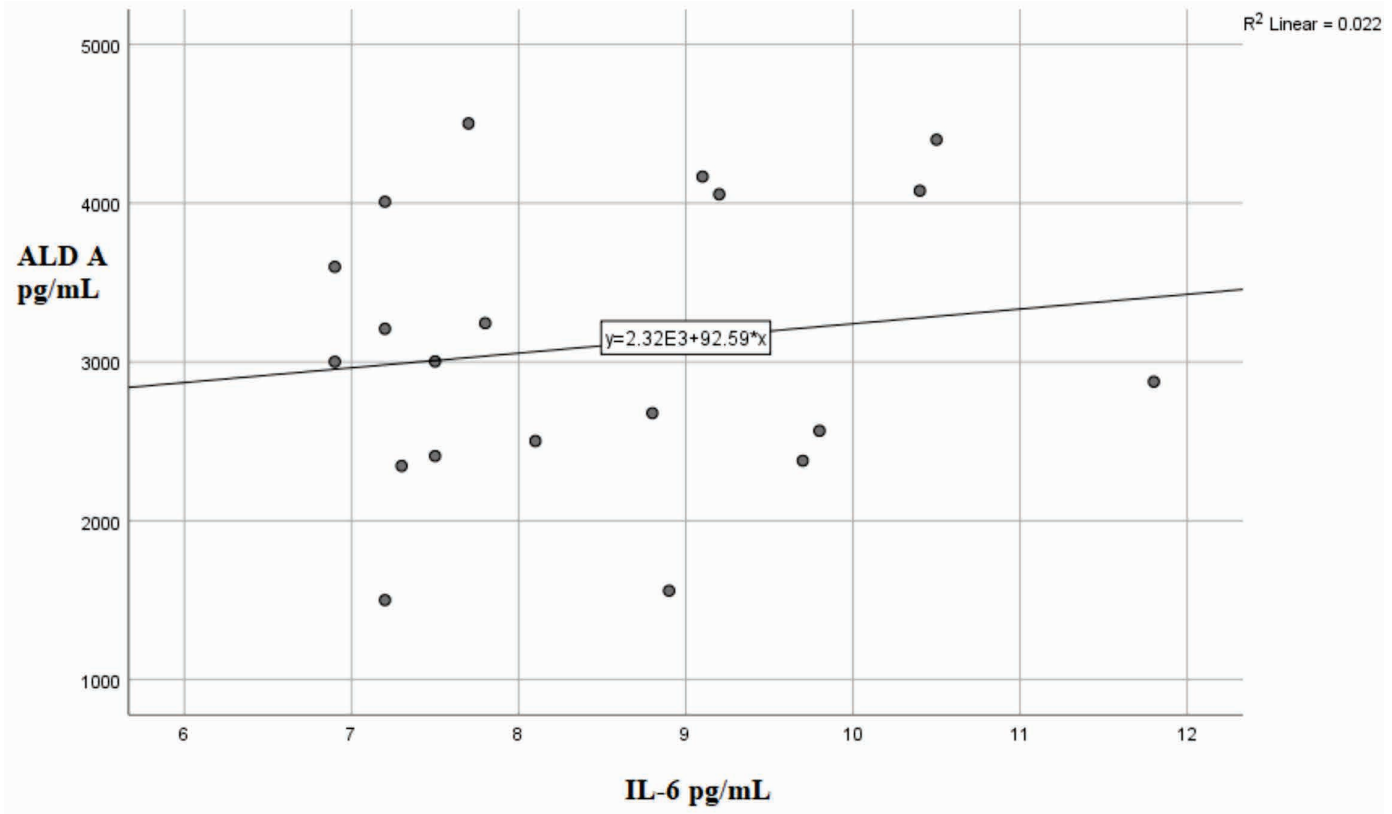

FIGURE 6. Correlation between aldolase A and IL-6 levels in the study group

TABLE 5. Correlation between ALD and IL-6 levels in patients with trauma

\begin{tabular}{|l|l|}
\hline Correlation & p* \\
\hline ALD A x IL-6 & $0.513, R=0.147$ \\
\hline
\end{tabular}

*Pearson correlation coefficient

The observed correlation between ALD A and IL- 6 was insignificant ( $p=0.513, R=0.147$ ), so no significant association was found between these two variables in patients with crushing syndrome.
TABLE 6. Correlation between aldolase $A$ and CK levels in crushing syndrome patients

\begin{tabular}{|l|l|}
\hline Correlation & $\mathbf{p}^{*}$ \\
\hline ALD A x CK & $0.829, R=0.092$ \\
\hline
\end{tabular}

*Pearson correlation coefficient

\section{DISCUSSIONS}

Crush syndrome can cause some severe life-threatening complications such multiple organ dysfunction 


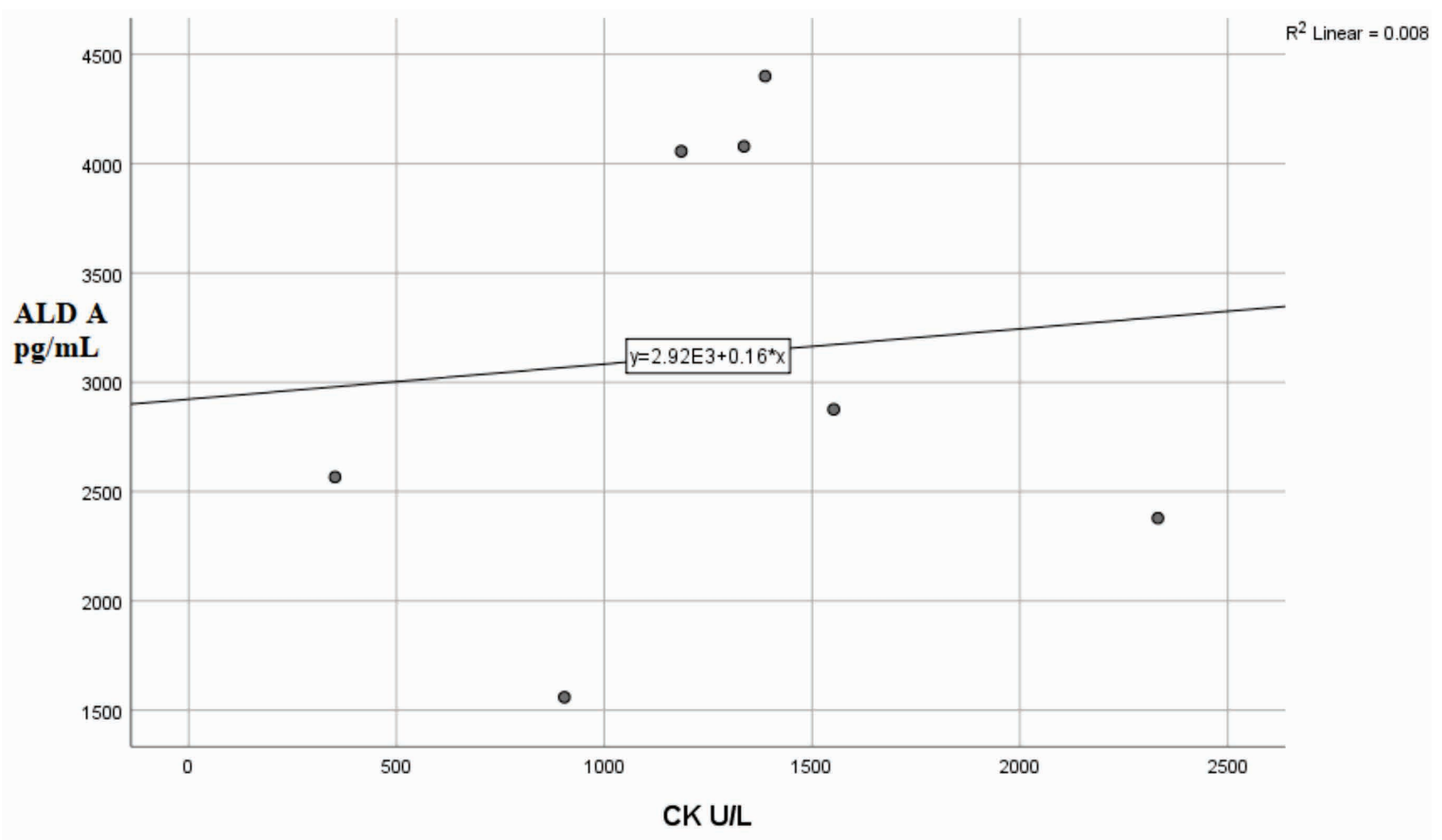

FIGURE 7. Correlation between aldolase $A$ and CK levels in the study group

syndrome, acute kidney injury and acute compartment syndrome, disseminated intravascular coagulation and severe arrhythmia.

Diagnosis of muscle crush injury is usually made through the correlation of clinical symptoms, by using a variety of imagining methods (such as $\mathrm{CT}, \mathrm{MRI}$ and ultrasonography), or by using some classical blood markers such C-reactive protein (CRP), creatine kinase-muscle (CK-MM), myoglobin, troponin, some species of interleukins, lactate dehydrogenase (LDH), aspartate aminotransferase (AST), fibrinogen, none of them being a specific biomarker for musculoskeletal injuries [57].

So far, in the medical literature, there are very few publications that associate the occurrence of crushing syndrome with changes in ALD A and CA III values.

ALD $A$ is an enzyme expressed in a variety of tissues, the most representative being the muscle. ALD A levels are elevated in response to skeletal muscle disease, polymyositis, dermatomyositis, muscular dystrophy, and inflammatory muscle disease [8]. ALD A has been studied primarily in the context of osteoarticular diseases (OAD) and compared with established markers of muscle destruction and inflammation- CK- MM, CRP.

ALD A has been shown to be a more sensitive prognostic factor for skeletal muscle damage in patients with OAD than the other two markers, CRP and CK$M M$, and the results obtained were very significant $(p<$ 0.001 ) for all markers. Our data clearly indicated the presence of inflammation, which was confirmed by the CRP test and established skeletal muscle damage, by the presence of elevated levels of ALD A. CK-MM levels were also increased along with the other two biomarkers, indicating that muscle degeneration occurred in patients with OAD [8-11].

Therefore, the elevated levels of CRP, CK-MM and ALD A observed in patients with OAD confirmed that these biomarkers are predictive risk factors that can be monitored and serve as one of the best rapid and lowcost diagnostic protocols, accessible even in the early progressive stage of OAD [12].

CAlll has been shown to be decreased in several diseases related to fatigue and muscle pain, such as rheumatoid arthritis and systemic lupus erythematosus [13]. Serum level variation of this parameter was reported in some diseases, such as rheumatoid arthritis, systemic lupus erythematosus, myasthenia gravis, but also after extensive physical activity that could lead to muscle fatigability and amyosthenia $[13,14]$.

The aim of our study was to correlate the changes of ALD A and CA III in the appearance and evolution of the crushing syndrome. Starting from the premise that ALD A and CA III undergo significant changes in the context of chronic osteoarticular diseases, our research team wanted to expand the research area of these two markers in traumatic pathology.

The data obtained in our study showed significant increases of the two markers (ALD A and CA III). These results encourage us to continue in the future the research of these two markers (ALD A and CAIII) on a larger number of patients with crushing syndrome. The in- 
creased specificity of these markers in the rapid diagnosis of crushing syndrome may contribute to the development of an early diagnostic protocol. This can lead to an increase in the survival rate of patients and a decrease in the occurrence of local and general complications, increasing the life expectancy of patients with crushing syndrome.

\section{CONCLUSION}

Although our study is at the beginning and the number of patients is low, the results obtained are very promising. The significantly increased levels of ALD A and CA III in crushing syndrome patients suggests that these two markers may become an important part in the rapid diagnosis of the crushing syndrome in the future.
Conflict of interest: none declared Financial support: none declared

\section{REFERENCES}

1. Plebani M. Skeletal muscle biomarkers: not new but still interesting diagnostic tools. Clin Chem Lab Med. 2010 Jun;48(6):745-6.

2. Shi C, Uda Y, Dedic C, Azab E, Sun N, et al. Carbonic anhydrase III protects osteocytes from oxidative stress. FASEB J. 2018 Jan;32(1):440-452.

3. Shang X, Chen S, Ren H, Li Y, Huang H. Carbonic anhydrase III: the new hope for the elimination of exercise-induced muscle fatigue. Med Hypotheses. 2009 Apr;72(4):427-9.

4. Liu M, Walter GA, Pathare NC, Forster RE, Vandenborne K. A quantitative study of bioenergetics in skeletal muscle lacking carbonic anhydrase III using 31P magnetic resonance spectroscopy. Proc Natl Acad Sci U S A. 2007 Jan 2;104(1):371-6.

5. Zhang CD, Lv FQ, Li QY, Zhang Y, Shi XQ, Li XY, Tang J. Application of contrastenhanced ultrasonography in the diagnosis of skeletal muscle crush injury in rabbits. $\mathrm{Br}$ J Radiol. 2014 Sep;87(1041):20140421.

6. Casciola-Rosen L, Hall JC, Mammen AL, Christopher-Stine L, Rosen A. Isolated elevation of aldolase in the serum of myositis patients: a potential biomarker of damaged early regenerating muscle cells. Clin Exp Rheumatol. 2012 Jul-Aug;30(4):548-53.

7. Casciola-Rosen L, Nagaraju K, Plotz $P$, Wang K, Levine S, Gabrielson E, Corse A, Rosen A. Enhanced autoantigen expression in regenerating muscle cells in idiopathic inflammatory myopathy. J Exp Med. 2005 Feb 21;201(4):591-601.

8. Ukaji F, Kitajima I, Kubo T, Shimizu C, Nakajima T, Maruyama I. Serum samples of patients with rheumatoid arthritis contain a specific autoantibody to "denatured" aldolase $\mathrm{A}$ in the osteoblast-like cell line, MG-63. Ann Rheum Dis. 1999 Mar;58(3):169-74.

9. Laureys M, Sion JP, Slabbynck H, Steenssens L, Cobbaert C, Derde MP, Gorus FK. Macromolecular creatine kinase type 1: a serum marker associated with disease. Clin Chem. 1991 Mar;37(3):430-4.

10. Galarraga B, Sinclair D, Fahie-Wilson MN, McCrae FC, Hull RG, Ledingham JM. A rare but important cause for a raised serum creatine kinase concentration: two case reports and a literature review. Rheumatology (Oxford). 2003 Jan;42(1):186-8.
11. Smith JW, Martins TB, Gopez E, Johnson T, Hill HR, Rosenberg TD. Significance of C-reactive protein in osteoarthritis and total knee arthroplasty outcomes. Ther $A d v$ Musculoskelet Dis. 2012 Oct;4(5):315-25.

12. Liu C, Wei Y, Wang J, Pi L, Huang J, Wang P. Carbonic anhydrases III and IV autoantibodies in rheumatoid arthritis, systemic lupus erythematosus, diabetes, hypertensive renal disease, and heart failure. Clin Dev Immunol. 2012;2012:354594.

13. Shaikh AB, Fang H, Li M, Chen S, Shang P, Shang $X$. Reduced expression of carbonic anhydrase III in skeletal muscles could be linked to muscle fatigue: A rat muscle fatigue model. J Orthop Translat. 2019 Sep 23;22:116-123.

14. Xiliang S, Shiyi C, Huimin R, Yunxia L, He H, Jian Z. Effects of Acute Intense Treadmill Running and Low-frequency Electric Stimulation on the Expression of CA-Protein in Skeletal Muscles and Sera of Rats. Chin J Sports Med. 2009;28(2):158-161. 